\title{
Mycobacterium tuberculosis Bacteremia Diagnosed in an HIV-Negative Patient in Brazil: A Rare or an Under-Reported Event?
}

\author{
D. J. Hadad ${ }^{1}$, A.C.C. Pignatari ${ }^{2}$, \\ M.A.O. Machado ${ }^{2}$ and M.A.S. Telles ${ }^{3}$
}

\author{
Centre of Infectious Diseases, Biomedical Center, Espirito Santo Federal \\ Universityl, Vitória, ES; Infectious Diseases Division, São Paulo Federal \\ University $^{2}$, São Paulo; Adolpho Lutz Institute ${ }^{3}$, São Paulo,SP, Brazil
}

\begin{abstract}
A case of Mycobacterium tuberculosis bacteremia in an HIV negative immunodepressed patient was described using the BACTEC 460 TB system. This bacterium should be investigated in the blood of immunodepressed non-HIV infected patients with prolonged fever. Key-Words: Mycobacterium tuberculosis, mycobacteremia, immunodeficiency.
\end{abstract}

On November 19 1998, a 34-year old white male was admitted to the emergency ward of São Paulo Hospital (Federal University of São Paulo, São Paulo, Brazil), complaining of a week of fatigue, with fever and rigors. He was born in Vitória da Conquista, BA, Brazil, worked as a brick layer, smoked cigarettes and drank alcohol. He denied any intravenous drug use, homosexual relations, or blood transfusions.

His past medical history included diagnoses of myelofibrosis and hepatosplenic schistosomiasis in February, 1993. Myelofibrosis was treated initially with prednisone (40 mg/d). In March, 1993, diabetes mellitus secondary to the use of corticosteroid was diagnosed and treated with insulin. In August, 1993, myelofibrosis was again treated with erythropoietin. Prednisone was gradually reduced and definitely interrupted in September 1993. Thereafter, no other drug was offered to treat the myelofibrosis. In February 1995, ELISA for HIV antibody was non-reacting. Upon initial examination, the patient was acutely ill, with hepatosplenomegaly and cervical lymphadenopathy;

Received on 29 October 2003; revised 27 February 2004.

Address for correspondence: Dr. David Jamil Hadad. Núcleo de Doenças Infecciosas/Centro Biomédico - Universidade Federal do Espírito Santo - Avenida Marechal Campos, 1468 Maruípe - Vitória-ES - Brasil - email: davhadad@ndi.ufes.br. Zip code: 29040-091.Phone: 027-3335-7208. Fax: 027-3335-7206.

The Brazilian Journal of Infectious Diseases 2004;8(2):184-185 (C) 2004 by The Brazilian Journal of Infectious Diseases and Contexto Publishing. All rights reserved. initial chest $\mathrm{X}$-ray showed diffuse interstitial infiltration, and subsequent right pleural effusion. His laboratory exams showed a hematocrit of $40 \%$, a white blood cell count of $1,700 \quad(5 \%$ blasts, $50 \%$ polymorphonuclear, $42 \%$ lymphocytes, $3 \%$ monocytes), and a platelet count of 39,000. No microorganisms were isolated from his blood or urine cultures in routine bacteriology; ELISA for HIV antibody was also non-reactive.

A few hours after admission, the patient developed seizures. On November 25, 1998, bronchoscopy with bronchoalveolar lavage (BAL) was performed. Over the next few days the patient developed hepatic encephalopathy. On December 11 1998, $5 \mathrm{~mL}$ of blood was inoculated into a BACTEC 13A vial and incubated in a BACTEC 460 TB system (Becton Dickinson); presumptive treatment for tuberculosis was then begun with streptomycin, ethambutol and ofloxacin due to liver failure. On December 13 1998, the patient died. No necropsy was performed. On January 27, 1999 acidfast bacilli (AFB) were detected and later identified as M. tuberculosis, sensitive to rifampin, isoniazid and ethambutol (pyrazinamide was not tested). Cultures of BAL and pleural fluid were negative for $M$. tuberculosis.

Mycobacterium tuberculosis (M. tuberculosis) bacteremia has become a common complication among HIV-infected patients, particularly in developing and under-developed countries [1,2]. However there are very few cases described in the literature in patients 
who are not infected by human immunodeficiency virus (HIV) [3].

This case report clearly shows the possibility of detecting $M$. tuberculosis bacteremia in a non-HIV infected patient using contemporary automated culture systems, such as the BACTEC 13A vial (BACTEC $460 \mathrm{~TB})$. In spite of his negative HIV sorology, myelofibrosis and hepatosplenic schistosomiasis probably contributed to $M$. tuberculosis isolation from blood. The authors point out the possibility of isolating M. tuberculosis from blood during the investigation of prolonged fever in other immunodepressed non-HIV infected patients, particularly in areas endemic for tuberculosis, like Latin America and Africa. The question of whether $M$. tuberculosis bacteremia in HIV negative patients is a rare or under-reported event could be answered in well-controlled, prospective studies.

\section{References}

1. Gilks C.F., Brindle R.J., et al. Extrapulmonary and disseminated Tbc in HIV-1 seropositive patients presenting to the acute medical Services in Nairobi. AIDS 1990;4(10):981-5.

2. Bouza E., Diaz-Lopez M.D., Moreno S., et al. Mycobacterium tuberculosis bacteremia in patients with and without human immunodeficiency virus infection. Arch Intern Med 1993; $153: 496-500$.

3. Archibald L.K., Den Dulk M.O., Pallangyo K.J., Reller L.B. Fatal Mycobacterium tuberculosis bloodstream infections in febrile hospitalized adults in Dar es Salaam, Tanzania. Clin Infect Dis 1998;26:290-6. 